\title{
Anchusa crispa subsp. valincoana (Boraginaceae), une nouvelle sous-espèce endémique de Corse
}

\author{
Guilhan Paradis, Carole Piazza \& Yann Quilichini
}

\begin{abstract}
PARADIS, G., C. PIAZZA \& Y. QUILICHINI (2018). Anchusa crispa subsp. valincoana Paradis, Piazza \& Quilichini (Boraginaceae), a new subspecies endemic to Corsica. Candollea 73: 201-207. In French, English and French abstracts. DOI: http://dx.doi.org/10.15553/c2018v732a4

Anchusa crispa subsp. valincoana Paradis, Piazza \& Quilichini (Boraginaceae), a new endemic subspecies of the littoral of the western coast of Corsica is described and illustrated. It includes four sub-populations around the Gulf of Valinco, near Propriano. It differs by several morphological characters from Anchusa crispa Viv. subsp. crispa, present on two sites of the Corsican east coast, south of Solenzara and in several localities of the north-west coast of Sardinia, notably by its reddish flowering stems, subglabrous lower surface of leaves, bracts with subglabrous upper surface and glabrous below and its flowers with dark blue corolla. It also differs from Anchusa crispa subsp. maritima (Vals.) Selvi \& Bigazzi, a Sardinian endemic, located on some sites of the north coast of Sardinia, a taxon with brownish or greenish flowering stems, linear leaves and bracts with upper surface minutely pubescent.
\end{abstract}

\section{Résumé}

PARADIS, G., C. PIAZZA \& Y. QUILICHINI (2018). Anchusa crispa subsp. valincoana (Boraginaceae), une nouvelle sous-espèce endémique de Corse. Candollea 73: 201-207. En français, résumés anglais et français. DOI: http://dx.doi.org/10.15553/ c2018v732a4

Anchusa crispa subsp. valincoana Paradis, Piazza \& Quilichini (Boraginaceae), une nouvelle sous-espèce endémique du littoral de la côte occidentale corse, est décrite et illustrée. Elle comprend quatre sous-populations sur le pourtour du golfe de Valinco, près de Propriano. Elle diffère par plusieurs caractères morphologiques de Anchusa crispa Viv. subsp. crispa, présente sur deux sites de la côte orientale corse au sud de Solenzara et dans plusieurs localités du nord-ouest de la Sardaigne, notamment des tiges florifères rougeâtres, des feuilles à face inférieure subglabre, des bractées à face inférieure glabre et à face supérieure subglabre et par des fleurs à corolle bleu foncé. Elle diffère aussi de Anchusa crispa subsp. maritima (Vals.) Selvi \& Bigazzi, endémique sarde, localisée sur quelques sites de la côte nord de la Sardaigne, celle-ci présentant des tiges florifères brunâtres ou verdâtres, des feuilles linéaires et des bractées à face supérieure pubérulente.

\section{Keywords}

BORAGINACEAE - Anchusa - Corsica - New subspecies - Taxonomy

\footnotetext{
Addresses of the authors:

GP: 7, Cours Général Leclerc, 20000 Ajaccio, France. E-mail: guilhan.paradis@orange.fr

CP: Conservatoire Botanique National de Corse, Office de l'Environnement de la Corse, 20250 Corte, France.

YQ: UMR SPE 6134, CNRS - Université de Corse, 20250 Corte, France.

Submitted on October 30, 2017. Accepted on March 5, 2018.
}

First published online on November 5, 2018 


\section{Introduction}

Anchusa crispa Viv. (Boraginaceae) est une espèce protégée en France depuis 1982, peu fréquente et en danger d'extinction (Danton \& Baffray, 1995; Delage \& Hugot, 2015), endémique de quelques sites littoraux sableux du sud de la Corse et du nord-ouest de la Sardaigne (Selvi \& BigAzZI, 1998; Valsecchi, 1988; Bacchetta et al., 2008).

En Corse, la population de $A$. crispa se répartit en deux ensembles (Thiebaud, 1988; Conrad et al., 1989; Paradis \& Piazza, 1989a, 1989b; Paradis, 1990, 1991; Quilichini, 2001). Un ensemble est localisé dans le golfe de Valinco (côte occidentale corse), avec quatre sous-populations présentes à Cala Piscona, Cappicciolu, Capu Laurosu et Portigliolo. Un second ensemble est localisé au sud de Solenzara (côte orientale corse), incluant trois sous-populations, deux à Favona et une à Cannella.

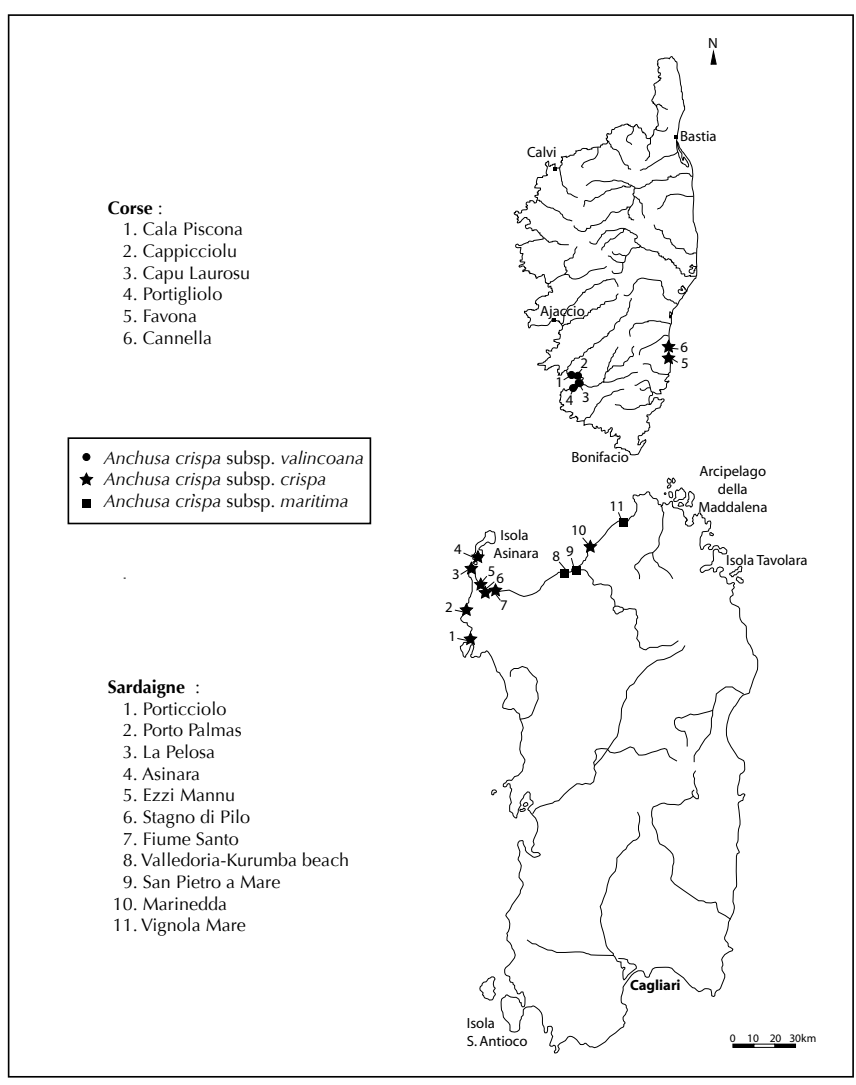

Fig. 1. - Carte de localisation des différentes sous-espèces de Anchusa crispa Viv. de la Corse et de la Sardaigne.

En Sardaigne, d'après Selvi \& Bigazzi (1998), deux sous-espèces sont présentes: $A$. crispa Viv. subsp. crispa, localisée à Marinedda, Foce di Fiume Santo, Stagno di Pilo, Ezzi Mannu, La Pelosa, Isola Asinara, Porto Palmas et Porticciolo et $A$. crispa subsp. maritima (Vals.) Selvi \& Bigazzi, localisée au nord-est de Castelsardo (fig. 1). D'après Coppi et al.
(2008) cette dernière n'est présente qu'en Sardaigne et leur travail montre une séparation génétique nette entre cette sousespèce et les autres populations sardes et corses de $A$. crispa. Au niveau morphologique, un port érigé et des feuilles linéaires permettent également de distinguer cette sous-espèce.

Les populations des côtes occidentale et orientale de la Corse sont très éloignées géographiquement (fig. 1) et aucun échange de gènes entre elles n'est naturellement possible. Quilichini et al. (2004) ont montré, à partir de l'analyse des isoenzymes, quelques différences chez des spécimens de A. crispa issus des deux côtes. Plus récemment, les analyses de la diversité génétique d'individus de Corse et de Sardaigne (Coppi et al., 2008) prouvent que les populations corses de Portigliolo (côte occidentale) et de Cannella (côte orientale) sont assez éloignées génétiquement l'une de l'autre, ce qui paraît aussi justifier la distinction de deux taxons. Ces deux types d'analyses, jointes aux grandes différences morphologiques, ne sont pas en accord avec la position d'une partie des auteurs italiens (Selvi \& Bigazzi, 1998; Bacchetta et al., 2008; Coppi et al., 2008), en opposition avec les chercheurs français, qui notent en Corse la seule présence d' $A$. crispa.

De plus, comme le montre cette étude, de nombreux caractères morphologiques permettent de les différencier. Aussi, nous proposons de distinguer deux taxons pour la Corse: (1) A. crispa subsp. crispa, sur la côte est, dont les caractères morphologiques sont semblables à ceux observés sur les populations sardes, (2) A. crispa subsp. valincoana Paradis, Piazza \& Quilichini, sur la côte ouest, taxon nouvellement décrit ici. Une telle vision taxonomique avait été d'ailleurs suggérée par Flora Corsica (Jeanmonod \& Gamisans, 2007, 2013) et Flora Gallica (Tison \& Foucault, 2014).

\section{Matériel et méthodes}

Les observations qualitatives et quantitatives des feuilles ont porté sur trente individus (appartenant à quatre sous-populations) de $A$. crispa subsp. valincoana et les mesures des calices et corolles des fleurs sur vingt-quatre individus. Pour $A$. crispa subsp. crispa, les mêmes observations qualitatives et quantitatives ont porté sur trente individus (appartenant à deux souspopulations) et les mesures des calices et corolles des fleurs sur vingt individus. Elles ont été réalisées sur le terrain et en laboratoire, sur des échantillons frais ou sub-frais (observés 1 ou 2 jours après leur prélèvement). Les photographies au microscope électronique à balayage (MEB) des bractées et des méricarpes ont été réalisées à l'Université de Corse.

L'étude comparative des deux sous-espèces de $A$. crispa s'est basée sur les caractères suivants: tiges (couleur, aspect de surface, degré de pilosité), feuilles des rosettes et des tiges floriferes (forme, face supérieure, face inférieure, bords, degré de pilosité), bractées de l'inflorescence (face supérieure, face inférieure, bords), pédicelle floral (couleur, longueur), calice de 
la fleur (couleur, forme, pilosité, longueur totale, longueur de la base), dimensions du calice fructifere, corolle de la fleur (couleur, diamètre, longueur du tube), longueur du style, caractères des cotylédons (longueur, largeur, pilosité), méricarpe (couleur, forme, dimensions, ornementation).

Pour étayer notre étude, un passage, en avril 2016, sur l'ensemble des populations sardes actuellement connues des deux sous-espèces de $A$. crispa a également été réalisé (Pinzza et al., 2017).

\section{Taxonomie}

Anchusa crispa subsp. valincoana Paradis, Piazza \& Quilichini, subspec. nova (fig. 1-3).

Holotypus: France. Corse: Golfe de Valinco, Cne de Propriano, près de Portigliolo, face à l'ancien restaurant

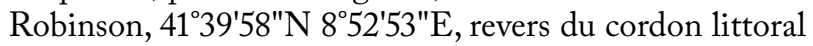
sableux, en avant des Tamarix africana, $5 \mathrm{~m}, 16 . \mathrm{IV} .2018$, Piazza 1804161 (Hb. CBNC!; iso-: G [G00341787]!, P!).

Anchusa crispa subsp. valincoana Paradis, Piazza $\xi^{\circ}$ Quilichini differs from its congeners by reddish stems, not very silky; upper side of the leaves dark green shiny, a little swollen on each side of narrowly grooved rib, with well-marked lobes, each carrying a few bristles; lower side of the leaves glabrous or with very little hair between the margin of the leaf blade and the median rib, the latter presenting little bristles; corolla dark blue to purple; cotyledons with bristly bairs.

Hémicryptophyte, pluriannuel ou bisannuel, en rosette(s), de 5-40 cm de hauteur, hispide dans toutes ses parties. Souche peu ou pas lignifiée, à racine pivotante, comportant 1 à 5 rosettes de feuilles généralement plaquées sur le substrat, parfois plus ou moins dressées, d'où naissent les tiges florifères. Tiges florifères en nombre variable par individu (de 3 à plus de 30), le plus souvent prostrées, parfois prostrées-ascendantes, rougeâtres, peu cannelées, hispides à nombreux poils de 1-1,5 mm, peu feuillées, de longueur variable $(7-30 \mathrm{~cm})$ en fonction des conditions de substrat, les plus longues ramifiées près de leur extrémité, chacune terminée par une inflorescence assez longue et lâche. Feuilles de la rosette oblongues, subsessiles, de 9 à $14 \mathrm{~cm}$ de long et de 1,5 à 2,5 cm de large; face supérieure vert foncé brillant, un peu renflée de part et d'autre de la nervure en rainure étroite, à lobes bien marqués, chacun portant quelques poils; face inférieure des feuilles quasi-glabre entre le bord du limbe et la nervure médiane, celle-ci présentant peu de poils. Feuilles caulinaires peu nombreuses (2-4), espacées, sessiles, embrassant un peu la tige, étroites $(0,5-1 \mathrm{~cm})$, de 4 à $8 \mathrm{~cm}$ de long. Inflorescences en cymes scorpioïdes, à nombre de fleurs variant en fonction de la teneur en eau du sable. Fleurs à pédicelle de $4-7 \mathrm{~mm}$, rougeâtre, à l'aiselle de bractées plus grandes que les calices ou les égalant. Bractées triangulairesovales, de (7-)10(-13) $\mathrm{mm}$; limbe de la face supérieure subglabre, à nervure médiane en rainure étroite; limbe de la face inférieure glabre, sauf dans la partie médiane présentant un alignement de poils; bordures des limbes portant de longs poils à base renflée, de près de $2 \mathrm{~mm}$ de long. Calice florifère hispide, long de 7-11 mm, comprenant un tube rougeâtre de 3-6 mm de long et 5 lobes arrondis, généralement verdâtres, le tube atteignant les deux tiers ou la moitié du calice. Calice fructifère de 8-10 mm de long sur 7-8 $\mathrm{mm}$ de large. Corolle actinomorphe, comprenant un tube blanc de $(3-) 4,5(-5) \mathrm{mm}$ de long et 5 lobes libres d'un bleu foncé ou violacé, de (7-)10(-11) $\mathrm{mm}$ de diam.; anneau de 5 grandes écailles blanches portant chacune de nombreux poils à leur extrémité. Androcée comportant 5 étamines, insérées sur le tube de la corolle, à anthères de 1,5-1,8 mm, un peu recouvertes par les écailles. Style de 4-5 mm de long; stigmate à 2 lobes ovoïdes, de $1,3 \times 2,1 \mathrm{~mm}$, tuberculés. Méricarpes grisâtres, ovoïdes, costulés, verruqueux, de 2,6-2,9 × 1,9-2,1 mm et à diamètre basal de 1,8-1,9 mm. Cotylédons à face supérieure hispide, à nombreux poils de $0,5 \mathrm{~mm}$.

Étymologie. - L'épithète valincoana se réfère au golfe de Valinco (côte occidentale corse), car cette sous-espèce est une micro-endémique corse, localisée actuellement sur quatre sites sableux des pourtours de ce golfe (Cala Piscona, Cappicciolu, Capu Laurosu et cordon littoral de Portigliolo).

Distribution. - La distribution de A. crispa subsp. valincoana se trouve être uniquement au fond du golfe de Valinco, avec quatre sous-populations (Cala Piscona, Cappicciolu, Capu Laurosu, Portigliolo). Anchusa crispa subsp. crispa n'est localisée elle qu'au sud de Solenzara, à Cannella et à Favona (avec deux sous-populations) (fig. 1).

Notes. - Le caractère le plus discriminant de $A$. crispa subsp. valincoana par rapport à la subsp. crispa et la subsp. maritima est l'aspect rougeâtre de ses tiges (tableau 1).

Anchusa crispa subsp. valincoana diffère de la subsp. crispa par les caractères suivants: (1) des tiges rougeâtres, peu poilues; (2) des fleurs à corolle bleu foncé à violet (fig. 2); (3) une face supérieure des feuilles vert foncé brillant, un peu renflée de part et d'autre de la nervure en rainure étroite, à lobes bien marqués, chacun portant quelques soies; (4) une face inférieure des feuilles glabre ou avec très peu de poils entre le bord du limbe et la nervure médiane, celle-ci présentant peu de poils (fig. 2); (5) des bractées à face supérieure subglabre, à face inférieure glabre sauf dans la partie médiane présentant un alignement de poils et à bord du limbe portant de longs poils espacés, à base renflée, de près de $2 \mathrm{~mm}$ de long (fig. 3).

Anchusa crispa subsp. maritima diffère de notre nouvelle sous-espèce par (1) un port érigé; (2) des feuilles caulinaires 
étroites; (3) des feuilles basales linéaires de 5 à $12 \mathrm{~mm}$ de large environ; (4) des bractées plus petites que le calice florifère et (5) un calice florifère divisé jusqu'au tiers et présentant des lobes arrondis (VAlsecchi, 1976, 1988; Selvi \& BigazZi, 1998).

\section{Clé des trois sous-espèces de Anchusa crispa}

1. Face supérieure des feuilles vert foncé brillant; tiges florifères rougeâtres à brunâtres 2

1a. Face supérieure des feuille vert clair mat; tiges florifères vert clair; feuilles basales généralement oblongues; face inférieure des feuilles pubescente, à poils très nombreux entre la nervure et les bords; corolle bleu clair
2. Tiges florifères rougeâtres; feuilles basales oblongues; face inférieure des feuilles quasi-glabre entre la nervure et les bords du limbe; corolle bleu foncé

subsp. valincoana

2a. Tiges florifères brunâtres, plus rarement vertes; feuilles basales linéaires; face inférieure des feuilles à poils peu nombreux entre la nervure et les bords; corolle bleu clair subsp. maritima subsp. crispa

Tableau 1. - Comparaison des principales différences des caractères morphologiques des trois sous-espèces de Anchusa crispa Viv. (subsp. crispa, subsp. valincoana Paradis, Piazza \& Quilichini et subsp. maritima (Vals.) Selvi \& Bigazzi).

\begin{tabular}{|c|c|c|c|c|}
\hline & & A. crispa subsp. crispa & A. crispa subsp. maritima & A. crispa subsp. valincoana \\
\hline \multirow{2}{*}{ Tiges } & Couleur et forme & vert clair; peu cannelée & brunâtre ou verdâtre; non cannelée & rougeâtre; parfois cannelée \\
\hline & Indument & hispide; trichomes courts $(0,5 \mathrm{~mm})$ & pubérulent; trichomes long ( $\geq 1 \mathrm{~mm}$ ) & hispide; trichomes long ( $\geq 1 \mathrm{~mm}$ ) \\
\hline \multirow{6}{*}{$\begin{array}{l}\text { Feuilles des } \\
\text { rosettes }\end{array}$} & Forme & obovale & linéaire, atténuée à la base & oblongue \\
\hline & $\mathrm{L}[\mathrm{cm}]$ & $10,3+/-3,66$ & $9-12$ & $11,69+/-2,62$ \\
\hline & I $[\mathrm{cm}]$ & $2,17+/-0,72$ & $0,5-1$ & $1,9+/-0,43$ \\
\hline & $\begin{array}{l}\text { Face supérieure } \\
{[\mathrm{mm}]}\end{array}$ & $\begin{array}{l}\text { limbe pubérulent, trichomes } \\
\text { env. } 1 \text {; nervure I = } 0,8\end{array}$ & $\begin{array}{l}\text { limbe subglabre; } \\
\text { nervure en rainure étroite, } I=<0,2\end{array}$ & $\begin{array}{l}\text { limbe subglabre; nervure } \\
\text { en rainure étroite, } 1=<0,2\end{array}$ \\
\hline & Face inférieure & $\begin{array}{l}\text { limbe pubescent; nervure } \\
\text { pubescente peu renflée }\end{array}$ & $\begin{array}{l}\text { limbe légèrement pubescent; } \\
\text { nervure pubescente peu renflée }\end{array}$ & $\begin{array}{l}\text { limbe subglabre; } \\
\text { nervure renflée pubérulente }\end{array}$ \\
\hline & Bords & quelques lobes peu marqués & aspect ondulé & $\begin{array}{l}\text { lobes assez marqués avec } \\
\text { quelques poils }\end{array}$ \\
\hline \multirow{4}{*}{$\begin{array}{l}\text { Bractées de } \\
\text { l'inflorescence }\end{array}$} & Forme de la base & $\begin{array}{l}\text { moyennement large, } \\
\text { plus grande que le calice }\end{array}$ & $\begin{array}{l}\text { moyennement large, } \\
\text { plus petite que le calice }\end{array}$ & $\begin{array}{l}\text { très large, plus grande } \\
\text { que le calice }\end{array}$ \\
\hline & Face supérieure & très pubescente & légèrement pubescent & subglabre \\
\hline & Face inférieure [mm] & pubescente, $L=0,5-2$ & glabre & glabre \\
\hline & Indument des bords & $\begin{array}{l}\text { nombreux poils, } \\
\text { en majorité courts }\end{array}$ & quelques poils longs & longs poils espacés \\
\hline \multirow{3}{*}{$\begin{array}{l}\text { Calice } \\
\text { de la fleur }\end{array}$} & Couleur et forme & $\begin{array}{l}\text { base un peu rosâtre; } \\
\text { lobes (sépales) subaigüs }\end{array}$ & $\begin{array}{l}\text { base rougeâtre; } \\
\text { lobes (sépales) arrondis }\end{array}$ & $\begin{array}{l}\text { base généralement rougeâtre; } \\
\text { lobes (sépales) lancéolés }\end{array}$ \\
\hline & $\begin{array}{l}\text { L moyenne totale } \\
{[\mathrm{mm}]}\end{array}$ & 5,6 (extrêmes: 5-6,2) & non mesuré & 8,48 (extrêmes: 7-11) \\
\hline & $\begin{array}{l}\text { L moyenne du tube } \\
\text { [mm] }\end{array}$ & 3,53 (extrêmes: 3-4,2) & non mesuré & 4,27 (extrêmes: 3-6) \\
\hline Pédicelle & Couleur & vert & brunâtre à vert & rougeâtre \\
\hline Calice fructifère & Taille $[\mathrm{mm}]$ & $7-8,2 \times 5-6$ & non mesuré & $8-10 \times 7-8$ \\
\hline \multirow{4}{*}{ Corolle } & Couleur des lobes & bleu très clair & bleu clair violacé à violet & bleu foncé ou un peu violet \\
\hline & Diamètre [mm] & 8,64 (extrêmes: 6,5-10) & non mesuré & 9,36 (extrêmes: 7-11) \\
\hline & L du tube $[\mathrm{mm}]$ & 4,73 (extrêmes: 4,1-5,2) & non mesuré & 5,06 (extrêmes: 4,5-5,5) \\
\hline & L/ & 1,28 & non mesuré & 1,75 \\
\hline
\end{tabular}




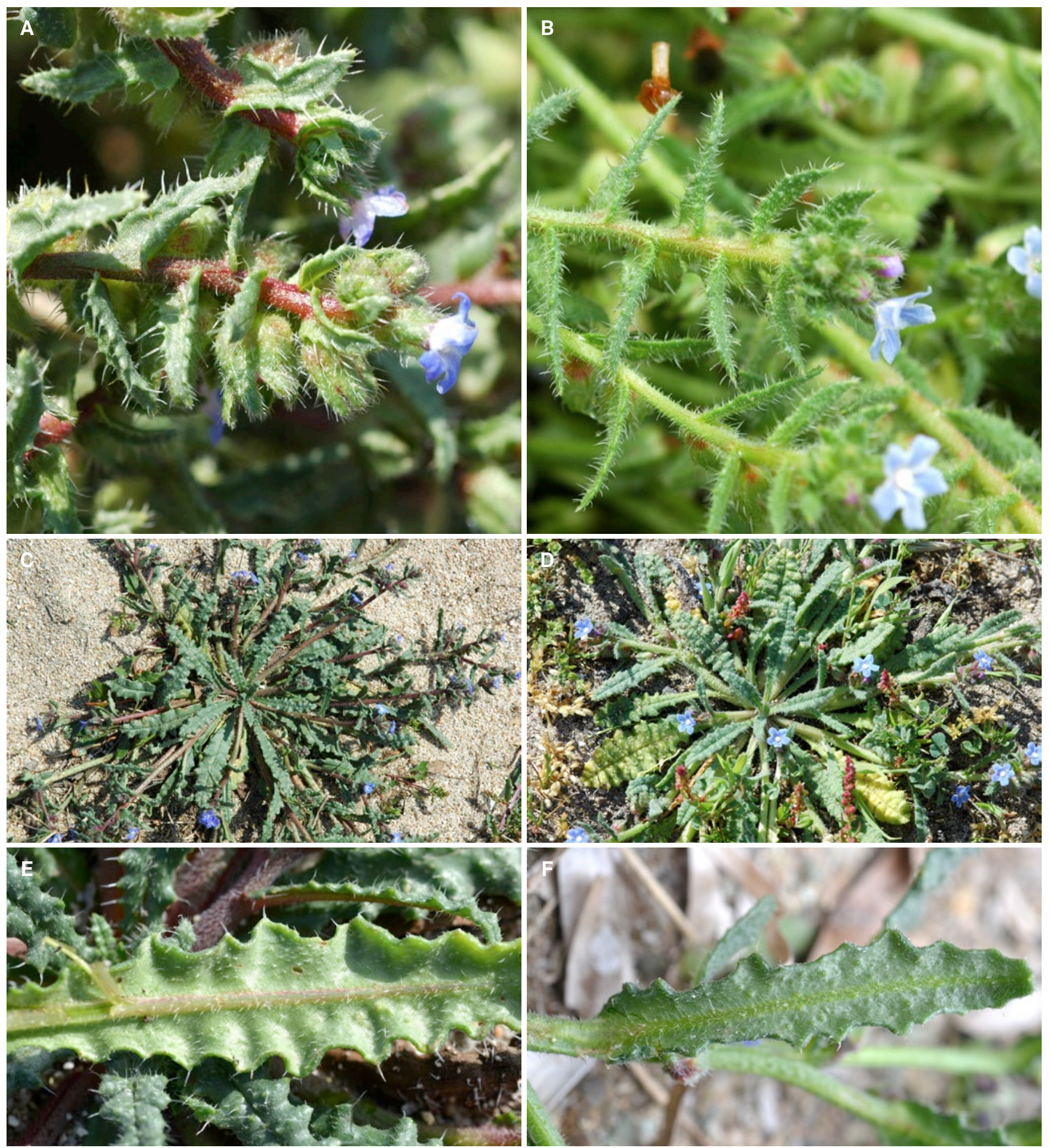

Fig. 2. - A. Anchusa crispa subsp. valincoana Paradis, Piazza \& Quilichini: tiges florifères; B. Anchusa crispa Viv. subsp. crispa: tiges florifères; C. Anchusa crispa subsp. valincoana: individus fleuris; D. Anchusa crispa subsp. crispa: individus fleuris;

E. Anchusa crispa subsp. valincoana: face inférieure d'une feuille; F. Anchusa crispa subsp. crispa: face inférieure d'une feuille.

[Photos: A, B: G. Paradis; C-F: C. Piazza] 

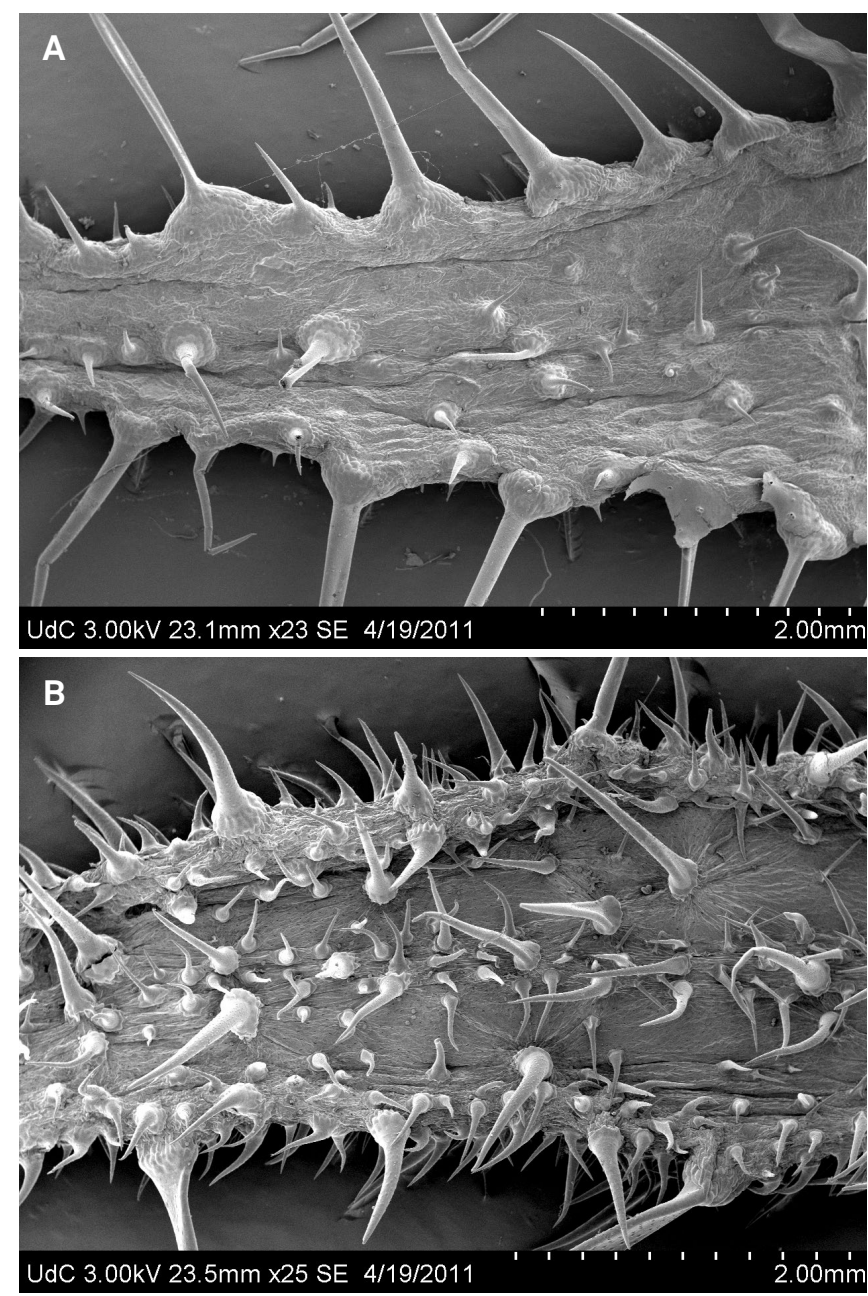

Fig. 3. - Photos au MEB de la face inférieure des bractées. A. Anchusa crispa subsp. valincoana Paradis, Piazza \& Quilichini; B. Anchusa crispa Viv. subsp. crispa. [Photos: Y. Quilichini]

Paratypi. - France. Corse: plage, embouchure de la Tavaria, 15.IV.1905, Rotgès, R. s.n. (P); Cala Piscona, cordon littoral, sables grossiers, $5 \mathrm{~m}$, 4.IV.2016, Piazza, C. s.n. (Hb. privé Piazza); Cappicciolo, cordon littoral, sables grossiers, alt. 6 m, 4.IV.2016, Piazza, C. s.n. (Hb. privé Piazza); Capu Laurosu, dépression au sud du cordon littoral, sables grossiers, 4 m, 9.II.2017, Paradis, G. s.n. (Hb. privé Paradis).

\section{Remerciements}

Nous remercions la DREAL de Corse qui a financé une partie de cette étude et nos homologues sardes: Prof. Emmanuele Farris, Prof. Stefania Pisanu et Arianna Amadori (Université de Sassari), Prof. Gianluigi Bacchetta (Université de Cagliari), Dr. Pierpaolo Congiatu (Directeur) et Dr. Giovanni Careddu (Parc National de l'Asinara), Prof. Antonio Farris (Président), Dr. Augusto Navone (Directeur) et Dr. Sergio Ortu (Parc Régional de Porto Conte) grâce à qui nous avons pu visi- ter l'ensemble des stations sardes de $A$. crispa. Nous sommes également très reconnaissants au Prof. Daniel Jeanmonod pour sa lecture critique d'une première version de ce manuscrit et à l'équipe éditoriale de Candollea pour leur expertise et la finalisation de cet article.

\section{Références}

Bacchetta, G., A. Coppi, C. Pontecorvo \& F. Selvi (2008). Systematics, phylogenetic relationships and conservation of the taxa of Anchusa (Boraginaceae) endemic to Sardinia (Italy). Syst. Biodivers. 6: 161-174.

Conrad, M., G. Paradis \& C. Piazza (1989). Anchusa crispa Viv. In: Jeanmonod, D. \& H.M. Burdet (ed.), Notes et contributions à la flore de Corse IV. Candollea 44: 385-386.

Coppi, A., A. Mengoni \& F. Selvi (2008). AFLP fingerprinting of Anchusa (Boraginaceae) in the Corso-Sardinian system: Genetic diversity, population differentiation and conservation priorities in an insular endemic group threatened with extinction. Biol. Conservation 141: 2000-2011.

Danton, P. \& M. BAfFray (1995). Inventaire des plantes protégées en France. Nathan.

Delage, A. \& L. Hugot (2015). Liste rouge régionale de la flore vasculaire de Corse. Conservatoire Botanique National de Corse, Office de l'Environnement de la Corse, Corte.

Jeanmonod, D. \& J. Gamisans (2007). Flora Corsica. Edisud.

Jeanmonod, D. \& J. Gamisans (2013). Flora Corsica. Ed. 2. Société botanique du Centre-Ouest, Jamac.

Paradis, G. (1990). Description de la végétation d'un site à Anchusa crispa Viv. sur la côte orientale de la Corse: l'Ouest de l'anse de Cannella. Doc. Phytosoc. 12: 189-201.

Paradis, G. (1991). Description de la végétation de quatre sites littoraux de la Corse orientale: Mucchiatana, Fautea, Pont de Fautea, Favona. Bull. Soc. Sci. Hist. Nat. Corse 661: 363-418.

Paradis, G. \& C. Piazza (1989a). Anchusa crispa Viv. à Capu Laurosu (Golfe de Valinco, Corse): localisations et rôles des bovins sur sa chorologie et sa biologie. Monde Pl. 436: 26-31.

Paradis, G. \& C. Piazza (1989b). Contribution à l'étude de la végétation du cordon littoral et de l'arrière-cordon de Portigliolo (Golfe de Valinco, Corse). Bull. Soc. Bot. Centre-Ouest ser. 2, 20: $51-75$.

Piazza, C., G. Paradis \& Y. Quilichini (2017). Anchusa crispa: caractérisation des taxons corses. Comparaison avec les taxons sardes. Rapport réalisé pour le compte de la DREAL de Corse.

Quilichini, A. (2001). Biologie et estimation des menaces pesant sur les populations de l'endémique corso-sarde Anchusa crispa Viv. (Boraginaceae). Acta Bot. Gallica 148: 319-331. 
Quilichini, A., M. Debussche \& J.D. Thompson (2004). Geographic differentiation of morphological traits and isozymes in the mediterranean island endemic Anchusa crispa: implications for the conservation of a protected species. Biol. Conservation 118: 651-660.

Selvi, F. \& M. Bigazzi (1998). Anchusa L. and allied genera (Boraginaceae) in Italy. Pl. Biosyst. 132: 113-142.

Thiebaud, M.A. (1988). Contribution à la connaissance de l'Anchusa crispa Viv. en Corse. In: Jeanmonod, D. \& H.M. Burdet (ed.), Notes et contributions à la flore de Corse, contribution 5. Candollea 43: 390-396.

Tison, J.-M. \& B. Foucault (2014). Flora Gallica. Flore de France. Ed. Biotope.

Valsecchi, F. (1976). Il genere Anchusa in Sardegna. Webbia 30: 43-68.

Valsecchi, F. (1988). Le piante endemiche della Sardegna. 190. Anchusa maritima Valsecchi, species nova. Boll. Soc. Sarda Sci. Nat. 26: 311-314. 